$\mathbb{T}$ periodica polytechnica

\author{
Transportation Engineering \\ $40 / 2(2012) 53$ \\ doi: $10.3311 / p p . t r .2012-2.02$ \\ web: http://www.pp.bme.hu/tr \\ (c) Periodica Polytechnica 2012
}

RESEARCH ARTICLE

\section{Modified Intelligent Driver Model}

\author{
Oussama Derbel / Tamás Péter / Hossni Zebiri / Benjamin Mourllion / Michel Basset
}

Received 2012-09-27

\begin{abstract}
The intelligent Driver Model (IDM) is an Adaptive Cruise Control (ACC) model which is wide used in transportation research. Although implementation simplicity in case of simulation, this model has drawbacks in terms of respect of the vehicle's capability and driver safety. This paper presents the stateof-the art of the IDM and the applied modifications to overcome its drawbacks.
\end{abstract}

\section{Keywords}

Intelligent Driver Model $\cdot$ Multiple vehicle systems $\cdot$ driver safety

\section{Acknowledgement}

The authors gratefully acknowledge the contribution of Agence Nationale de la Recherche within the Automatisation Basse Vitesse project ( $A B V$ project). The presented research work is sponsored by the OTKA CNK 78168 project, as well as the Control Engineering Research Group of the HAS at the Budapest University of Technology and Economics.TÁMOP4.2.2.C-11/1/KONV-2012-0012: "Smarter Transport" - IT for co-operative transport system - The Project is supported by the Hungarian Government and co-financed by the European Social Fund.

\section{Oussama Derbel}

MIPS Laboratory, EA 2332 Université de Haute-Alsace

12, rue des frères Lumière, 68093 Mulhouse Cedex, France

e-mail: oussama.derbel@uha.fr

\section{Tamás Péter}

Department of Control for Transportation and Vehicle Systems, BME, Stoczek J. u. 2., H-1111 Budapest, Hungary

e-mail: peter.tamas@mail.bme.hu

\section{Hossni Zebiri}

\section{Benjamin Mourllion}

MIPS Laboratory, EA 2332 Université de Haute-Alsace

12, rue des frères Lumière, 68093 Mulhouse Cedex, France

\section{Michel Basset}

MIPS Laboratory, EA 2332 Université de Haute-Alsace

12, rue des frères Lumière, 68093 Mulhouse Cedex, France

e-mail: michel.basset@uha.fr

\section{Notations}

$l_{n}$

$x_{n}$

$\dot{x}_{n}=v_{n}$

$\ddot{x}_{n}=\dot{v}_{n}$

$\Delta x_{n}=x_{n+1}-x_{n}$

$\Delta v_{n}=v_{n+1}-v_{n}$

$\Delta a_{n}=a_{n+1}-a_{n}$

$n^{\text {th }}$ vehicle length (m)

$n^{\text {th }}$ vehicle position (m)

$n^{\text {th }}$ vehicle velocity $\left(\mathrm{m} \cdot \mathrm{s}^{-1}\right)$

$n^{\text {th }}$ vehicle acceleration $\left(\mathrm{m} . \mathrm{s}^{-2}\right)$

bumper to bumper distance gap $(\mathrm{m})$

$n^{\text {th }}$ vehicle velocity difference $\left(\mathrm{m} . \mathrm{s}^{-1}\right)$

$n^{\text {th }}$ vehicle acceleration difference $\left(\mathrm{m} \cdot \mathrm{s}^{-2}\right)$

\section{Introduction}

The concept of controlling the vehicle speed dates back to 1788 ([4]). The first experience was made by James Watt and Matthew Boulton to control the speed of a stream engine [5, 6]. After that, researchers were continuing to develop speed control systems in many fields.

In the automobile field, during the last decade, Advanced Driver Assistance Systems (ADAS) have been developed and implemented by automotive manufacturer. Such ADAS systems like Anti lock Braking System (ABS) allows the wheels to continue interacting with the road surface and avoid skidding. These systems include, also, the Adaptive Cruise Control (ACC) system functionality. The first vehicle with Adaptive Cruise Control shipped in 1995. This system tries to reduce human driving task. Nowadays, car manufacturers, such as Audi, Volkswagen and Peugeot, are developing and improving their ACC system to adjust it to different traffic situations using vehicle sensors and actuators.

In 2000, the Transportation laboratory in technical university of Dresden in Germany published the Intelligent Driver Model (IDM) which was used by BMW car manufacturer. Compared to the other ACC model, the IDM has more advantages in terms of implementation, calibration and intuitive parameters. Nevertheless, the IDM has drawbacks. The goal of this presented work is to improve the IDM model to have more performance in terms of driver safety and of realistic response in case of critical situations such as collision.

In this paper, Section two presents the original formulation of the Intelligent Driver Model. Section three presents the state of the art of the Intelligent Driver Model. Section four presents 
the desired minimum gap modification for driver safety. Section five presents the modification of the Intelligent Driver Model related to the vehicle's real capability. Section six presents the full modified IDM. Section seven concludes and gives outlooks.

\section{Initial formulation of Intelligent Driver Model}

The Intelligent Driver Model (IDM) is an Adaptive Cruise Control model with which driver can preset his desired speed and safety time gap. This continuous model, presented by [15], is given by

$$
\dot{v}_{n}=a_{n} \cdot\left(1-\left(\frac{v_{n}}{v_{n}^{0}}\right)^{\delta}-\left(\frac{s^{*}\left(v_{n}, \Delta v_{n}\right)}{s_{n}}\right)^{2}\right),
$$

where $a_{n}$ is the maximum acceleration of the vehicle $n$ $\left(\mathrm{m} . \mathrm{s}^{-2}\right), v_{n}^{0}$ the desired velocity of the vehicle $n\left(\mathrm{~m} . \mathrm{s}^{-1}\right), \delta$ a parameter of model (According to [16], this parameter is almost fixed at 4$), s_{n}$ the net distance gap (m) defined as $s_{n}=\Delta x_{n}-l_{n+1}$ and $s_{n}^{*}$ the desired minimum gap of the vehicle $n$ given by

$$
s^{*}\left(v_{n}, \Delta v_{n}\right)=s_{n}^{0}+T_{n} v_{n}-\frac{v_{n} \Delta v_{n}}{2 \sqrt{a_{n} b_{n}}},
$$

where $b_{n}$ is the desired deceleration of the vehicle $n\left(\mathrm{~m} \cdot \mathrm{s}^{-2}\right)$, $s_{n}^{0}$ the jam distance of the vehicle $n(\mathrm{~m})$ and $T_{n}$ the safety time gap of the vehicle $n(\mathrm{~s})$.

\section{State-of-the-art of Intelligent Driver Model}

The Intelligent Driver Model, presented in the last Section, is wide used in transportation research area:

In the Multi-model Open-source Vehicular-traffic Simulator, [7] use the IDM to simulate the longitudinal vehicle motion. In addition, this simulator presents a lane changing strategy which consists in comparing acceleration costs. If this cost is greater than a settled threshold, the vehicle can change lane. Moreover, the IDM is implemented in other traffic simulators such as SUMO (Simulation of Urban Mobility) simulator and VISSIM (Verkehr In Städten - SIMulationsmodell:German for Traffic in cities - simulation mode).

[8] study the single lane traffic inhomogeneity using the IDM model. For different percentage of cars and tracks, traffic flow and vehicles density are calculated and compared to empirical data of extended congested traffic on the Dutch freeway A9. By analyzing the flow density diagram and the time series of singlelane heterogeneous traffic, results show the typical inverse $\lambda$ form with a distinct gap between free and congested traffic data. Furthermore, in this reference, characteristics between free and congested traffic are compared.

The parameter sensitivity according to the string stability of a platoon vehicle is made by [9]. Fig. 1, taken from this reference, shows the phase diagram of congested traffic states in the phase space spanned by the number $n_{a}$ of anticipated vehicles and the reaction time $T^{\prime}$ in the open system with a bottleneck as described. The dynamic phases homogeneous con- gested traffic (HCT), oscillatory congested traffic (OCT), triggered stop-and-go (TSG), and moving and pinned localized clusters (MLC/PLC) are discussed in the main text. In this figure, we see that when the reaction time is high and the spatial anticipation number is small, crashes will occur in case of critical situation.

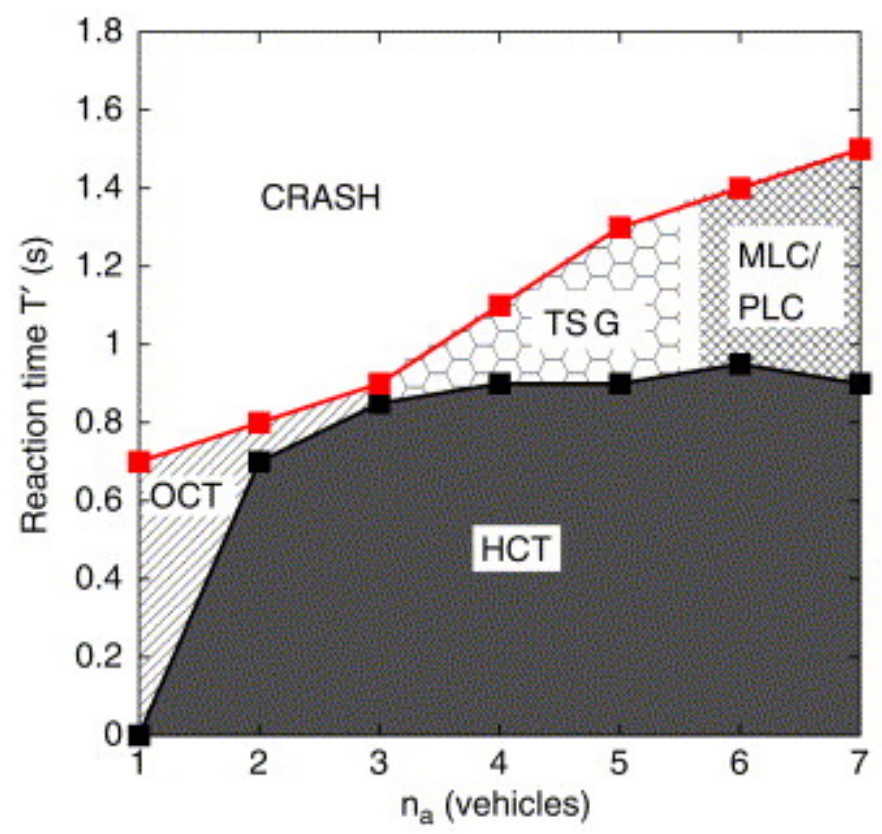

Fig. 1. Reaction time-spatial anticipation diagram $([8])$

[16] extended the IDM to human driver model by adjusting its parameters and adding noise. Impact of IDM equipped vehicle on traffic flow and travel time in Open system with a bottleneck are investigated. Results show that when the percentage of ACC vehicles increases traffic flow and travel time decrease.

[11] studied the convective instability in congested traffic flow using the Intelligent Driver Model. As he says in this reference: an extended open system such as traffic flow is said to be convectively unstable if perturbations of the stationary state grow but propagate in only one direction, so they eventually leave the system.

[12] uses the IDM model to study the impact of Adaptive Cruise Control on traffic flow. In his studies, the traffic is divided into clusters where vehicles motion is represented by the Intelligent Driver Model.

[10] use the Intelligent Driver Model as a basic example of a car-following model representing the operational level of driving in agents traffic simulation.

In the literature, there is, also driver safety studies using the IDM Model such as done by [13] in case of mixed traffic. This last denotes the coexistence of two driving styles: automated and manual driving. Automated vehicles are equipped by the Intelligent Driver Model. Figs. 2 and 3 shows the relative safety percentage as function as the percentage of IDM in the traffic in case of accident scenario. In this last, the platoon leader 
brakes suddenly and stops instantly after the platoon stabilization (catastrophic scenario). Results show that in the presence of more equipped vehicles with IDM, traffic is more safe in terms of collision number and collision severity.

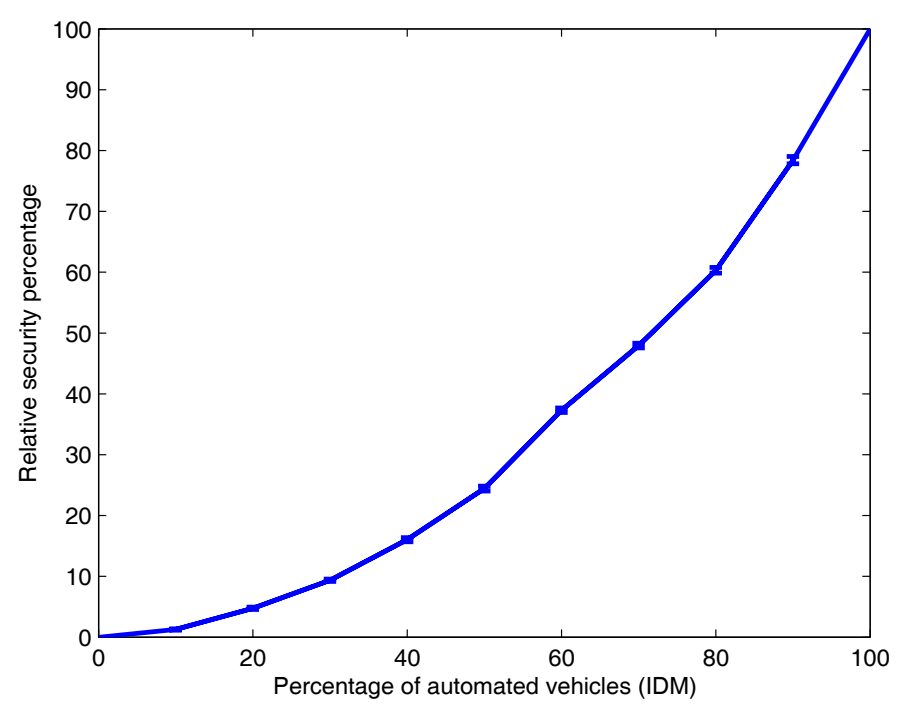

Fig. 2. Relative safety index based on the collision number ([14])

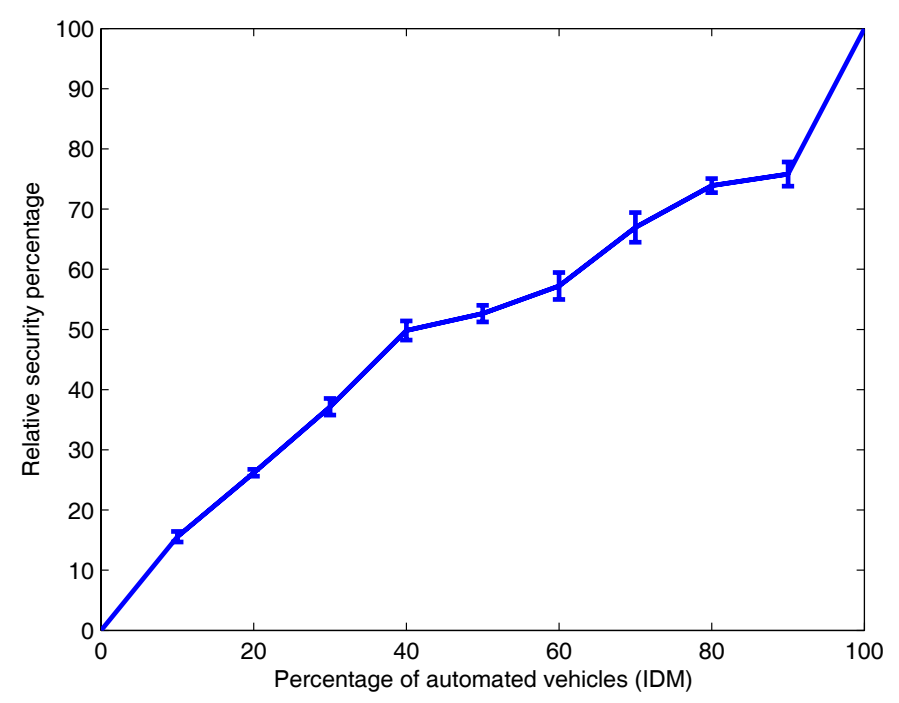

Fig. 3. Relative safety index based on the Equivalent Energy Speed

In case of collision free scenario, the Dwell Time safety indicator is used for safety evaluation in mixed traffic road. Fig. 4 shows that, at constant density, the driver safety increases when the percentage of automated vehicles, which are equipped with IDM, increases also. Furthermore, when the traffic density increases driver safety increases.

\section{Desired minimum gap modification of Intelligent Driver Model for driver safety}

\subsection{Modification}

Eq. (2) contains three terms:

The first one is the minimum distance $s^{0}$ in congested traffic.

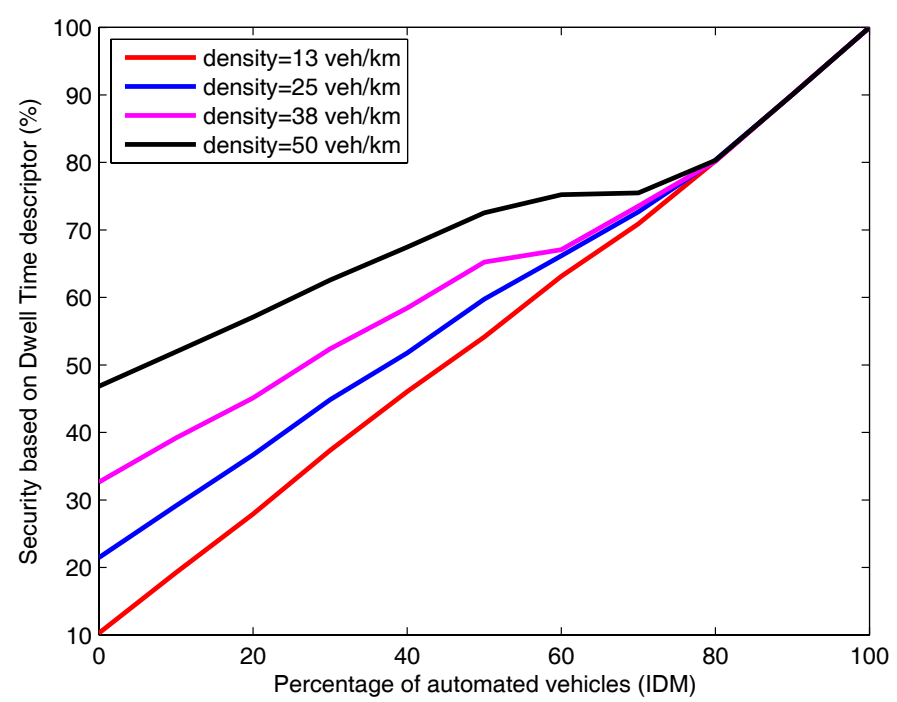

Fig. 4. Safety percentage in case of collision free scenario ([13] $)$

This term is significant only for low velocity.

The second term is $T_{n} v_{n}$ which corresponds to the safety gap that the follower must have with its leader. This term is active in stationary traffic. The last term, $-\frac{v_{n} \Delta v_{n}}{2 \sqrt{a_{n} b_{n}}}$, is only active in non-stationary traffic situation.

Because driver safety is not totally guaranteed by mean of the actual desired minimum gap in non stationary traffic, the term given by

$$
c_{n} \frac{v_{n}^{2}}{b_{n}}
$$

is added to desired minimum gap.

The new desired minimum gap is given by

$$
s^{*}\left(v_{n}, \Delta v_{n}\right)=s_{n}^{0}+T_{n} v_{n}+c_{n} \frac{v_{n}^{2}}{b_{n}}-\frac{v_{n} \Delta v_{n}}{2 \sqrt{a_{n} b_{n}}},
$$

The goal to modify the desired minimum gap is to improve driver safety by increasing the minimum gap in case of critical situations such as accident scenario. So, in case of high speed, the desired minimum gap increases and automatically driver safety. In case of low speed, the added term, given by Eq. (3), has no significant impact on driver safety. In the next paragraph, the minimum $c_{n}$ value will be calculated using a critical accident scenario.

\subsection{Determination of the variable $c_{n}$ \\ 4.2.1 Scenario}

In [14] present a state-of-the-art of accident scenarios such as emergency braking and Bricks Wall. In this paragraph, a new one will be presented. This scenario consists on the brutally switching from a speed to another lower than the first speed. This scenario seems to be similar to the emergency braking scenario but it is more aggressive. Fig. 5 presents this test scenario which is given by leader speed profile. 


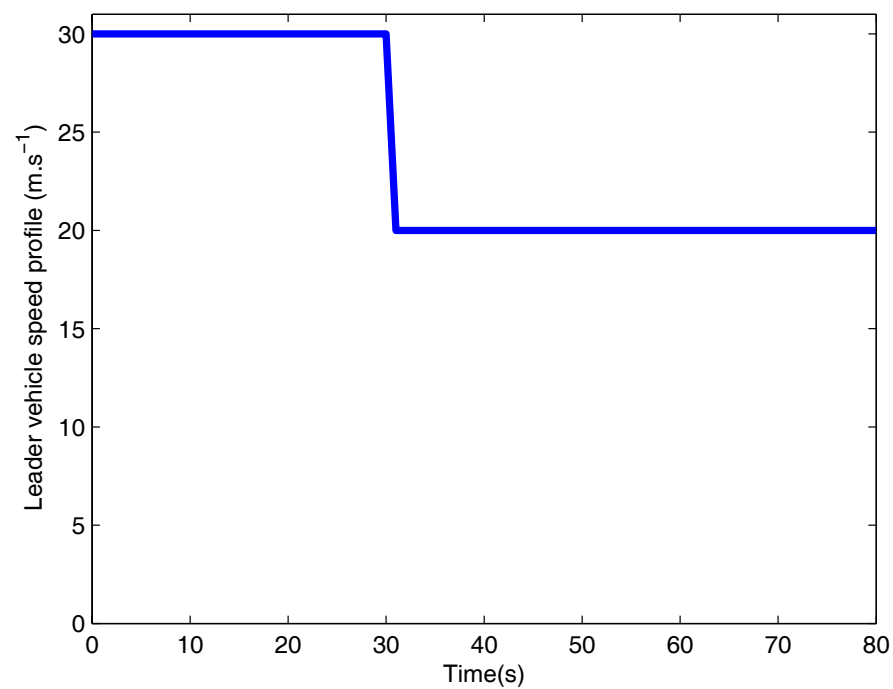

Fig. 5. Leader speed profile

\subsubsection{Safety descriptor}

Through the scenario described below, collision in the platoon will occur between follower vehicles. For this purpose, at constant density, number of collision will be the descriptor for traffic safety assessment and for $c_{n}$ determination. [13] define the relative safety indicator related to accident scenario as

$$
S_{\text {Coll }}=100 \frac{N b_{\text {Veh }}-N b_{\text {Crash }}}{N b_{\text {Veh }}}
$$

where $N b_{\text {Veh }}$ is the vehicle number in platoon, $N b_{\text {Crash }}$ the collision number and $S_{\text {Coll }}$ the relative safety index based on collision number.

\subsubsection{Simulation result}

The scenario described below will be applied in to simulation under IDM parameters given by table 1 .

Tab. 1. Intelligent Driver Model parameters

\begin{tabular}{llll}
\hline Parameter & mean value & Standard deviation & unit \\
\hline Maximum acceleration (a) & 6.0 & 0.3 & $\mathrm{~m} . \mathrm{s}^{-2}$ \\
\hline Desired deceleration (b) & 7.5 & 0.9 & $\mathrm{~m} . \mathrm{s}^{-2}$ \\
\hline Safety time gap (T) & 2.3 & 0.4 & $\mathrm{~s}$ \\
\hline Desired speed $\left(\mathrm{v}^{0}\right)$ & 30 & 3.0 & $\mathrm{~m} . \mathrm{s}^{-1}$ \\
\hline
\end{tabular}

Fig. 6 shows that when $c$ increases the driver safety increases also. This conclusion is evident since the desired minimum gap has increased with adding the positive term $c_{n} \frac{v_{n}^{2}}{b_{n}}$. In the other hand, this result shows that the safety time gap fixed by the driver before starting can be insufficient to avoid collision. In conclusion, with the new desired minimum gap, driver safety is more guaranteed even if the safety time gap,$T_{n}$, is not sufficient. In figure 6 to have a full safety driving, $c_{n}$ must be equal or greater than 0.4 . This value is the minimum one to have no collision in the platoon in case of critical accident scenario. To improve traffic flow, $c_{n}$ can be fixed at 0.4 since

$$
\left.s^{*}\left(v_{n}, \Delta v_{n}\right)\right|_{\left(c_{n}=0.4\right)}<\left.s^{*}\left(v_{n}, \Delta v_{n}\right)\right|_{\left(c_{n}>0.4\right)},
$$

then

$$
q_{\left(c_{n}=0.4\right)}>q_{\left(c_{n}>0.4\right)}
$$

where $q$ is the traffic flow. So, $c_{n}=0.4$ is the optimal value to improve safety and traffic flow at the same time.

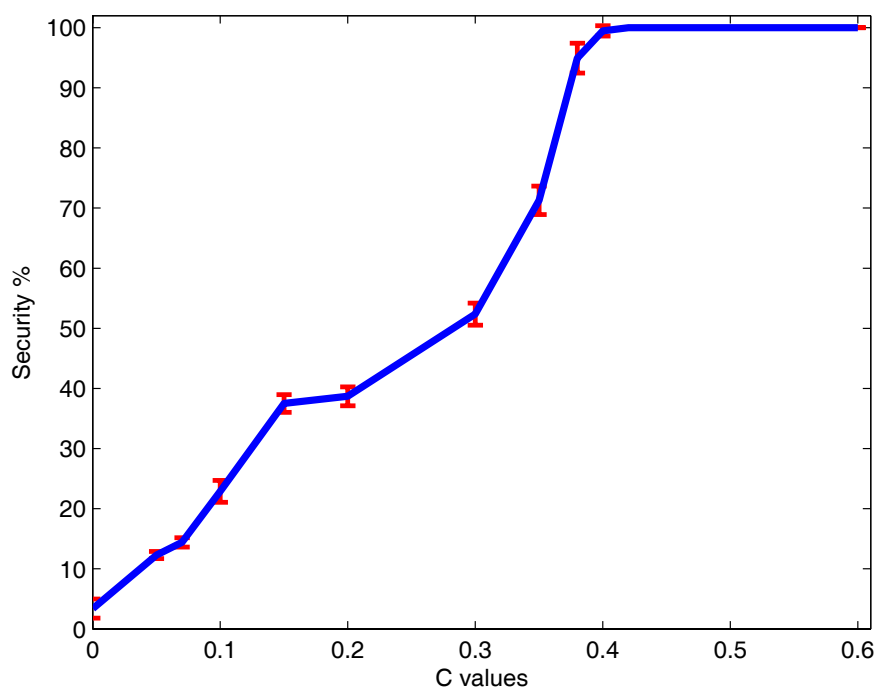

Fig. 6. Safety evaluation based on collision number as function as $c_{n}$ values

\section{Adaptation to the real vehicle capabilities}

\subsection{Formulation}

For a multi vehicle system, the Intelligent Driver Model is give by

$$
\langle\mathbf{A}\rangle^{-1} \ddot{\mathbf{x}}(t)+\langle\mathbf{V}\rangle^{-1} \mathbf{f}_{\mathbf{1}}(\dot{\mathbf{x}}(t))+\langle\mathbf{S}\rangle \mathbf{f}_{\mathbf{2}}(\mathbf{x}(t))=\mathbf{1}
$$

with

$$
\begin{aligned}
& \langle\mathbf{A}\rangle^{-1}=\left[\begin{array}{ccccc}
\frac{1}{a_{1}} & & & & \\
& \frac{1}{a_{2}} & & & \\
\vdots & \vdots & \vdots & \vdots & \vdots \\
& & & & \frac{1}{a_{n}}
\end{array}\right],
\end{aligned}
$$

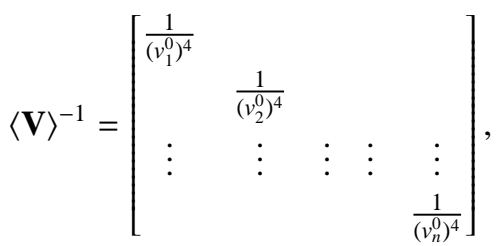

$$
\begin{aligned}
& \langle\mathbf{S}\rangle^{-1}=\left[\begin{array}{ccccc}
s_{1}^{2} & & & & \\
& s_{2}^{2} & & & \\
\vdots & \vdots & \vdots & \vdots & \vdots \\
& & & & s_{n}^{2}
\end{array}\right], \\
& \mathbf{f}_{\mathbf{1}}(\dot{\mathbf{x}}(t))=\left[\begin{array}{c}
\dot{x}_{1}^{4} \\
\dot{x}_{2}^{4} \\
\vdots \\
\dot{x}_{n}^{4}
\end{array}\right], \mathbf{f}_{\mathbf{2}}(\mathbf{x}(t))=\left[\begin{array}{c}
\frac{1}{\left(x_{0}-x_{1}\right)^{2}} \\
\frac{1}{\left(x_{1}-x_{2}\right)^{2}} \\
\vdots \\
\frac{1}{\left(x_{n-1}-x_{n}\right)^{2}}
\end{array}\right], \mathbf{1}(t)=\left[\begin{array}{c}
1(t) \\
1(t) \\
\vdots \\
1(t)
\end{array}\right]
\end{aligned}
$$




\subsection{First modification of the Intelligent Driver Model}

As shown in Eq. (6), there is the step function in the left side. It means that vehicles, which are stopped at initial time, start with no null acceleration. This drawback is caused by the non derivative and non continuity of the step function at time $t=0 \mathrm{~s}$. For this reason, this one can be replaced by a piecewise function given by

$$
E(x)=\left\{\begin{array}{lc}
0 & \text { if } t \leq 0 \\
\frac{t^{2}\left(t-2 \epsilon_{1}\right)^{2}}{\epsilon_{1}^{4}} & \text { if } 0<t \leq \epsilon_{1} \\
1 & \text { otherwise }
\end{array}\right.
$$

The E function is composed by two hyperbolic functions given by

$$
g_{1}: t \longmapsto t^{2} \quad g_{2}: t \longmapsto(t-2 \epsilon)^{2}
$$

The product $g_{1} g_{2}$ at time $t=\epsilon_{1}$ gives a result of $\epsilon_{1}^{4}$. To have 1 at time $t=\epsilon_{1} \mathrm{~s}$, the product is divided by $\epsilon_{1}^{4}$. Fig. 7 shows the E function with the example of $\epsilon_{1}=2 \mathrm{~s}$. With this function, the acceleration continuity is guaranteed and the initial acceleration at time $t=0 \mathrm{~s}$ is null. The $\epsilon_{1}$ is the response time of the ACC system. This parameter can be a driver comfort criterion also.

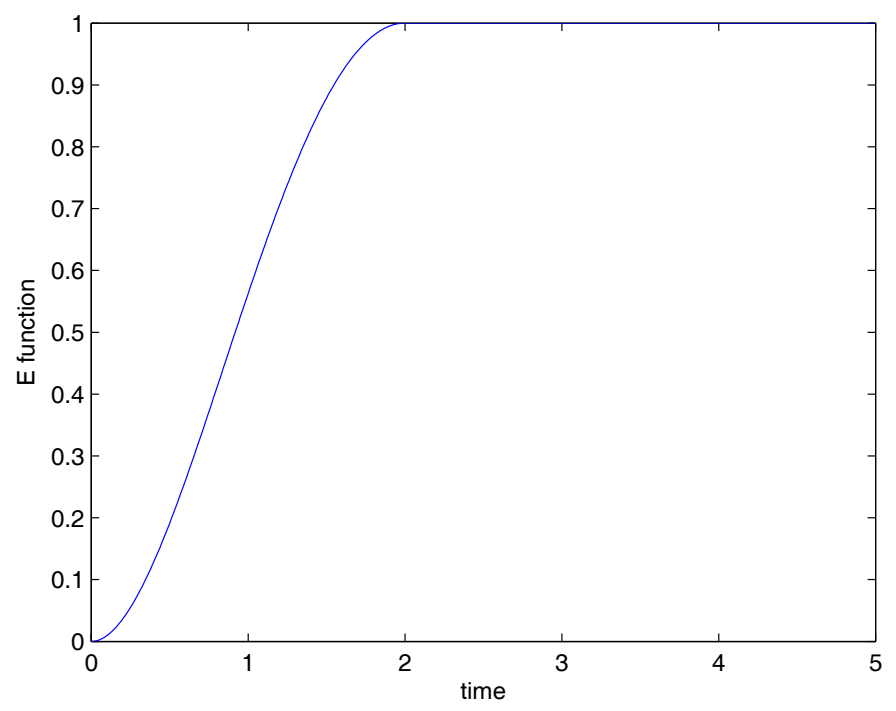

Fig. 7. E function $\left(\epsilon_{1}=2 \mathrm{~s}\right)$

\subsection{Second modification of the Intelligent Driver Model}

The principle of this new modification is to have the vehicle acceleration continuous and differentiable. As mentioned below, the IDM is collision free model. It means that the IDM generates a non realistic acceleration, which can exceeds the vehicle capability, when approaching to a vehicle ahead to avoid collision. Fig. 8 shows that the vehicle acceleration is infinite when the collision occurs. It is notified that the preceding results are made under saturation of the vehicle's real acceleration values.

This paragraph deals with the exceed of the vehicle deceleration just at collision time. Therefore, the $\mathbf{f}_{2}$ function, given by

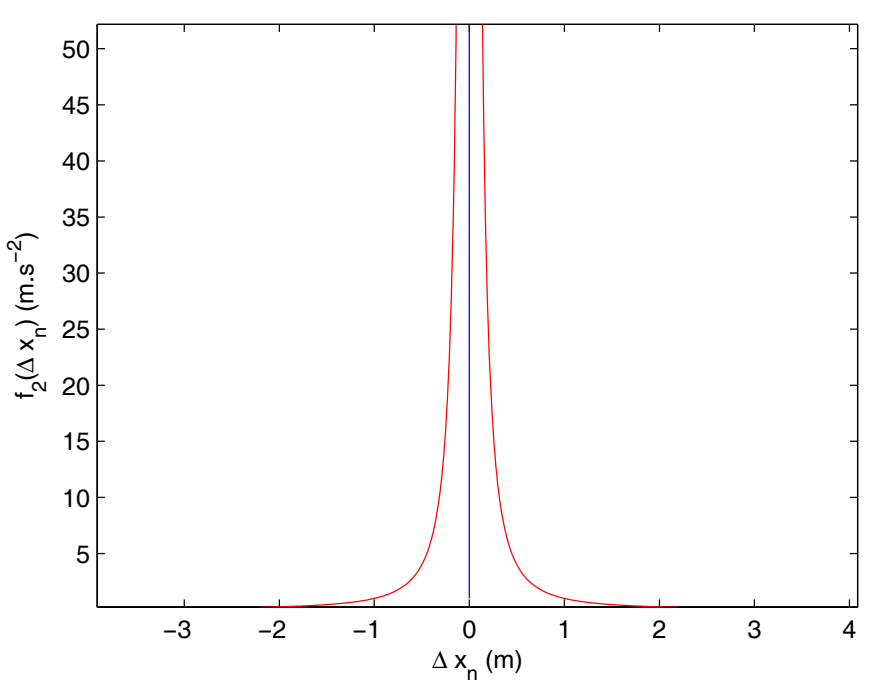

Fig. 8. Vehicle acceleration without the second modification in case of collision

$$
\mathbf{f}_{2}(\mathbf{x}(t))=\left[\begin{array}{c}
\frac{1}{\left(x_{0}-x_{1}\right)^{2}} \\
\frac{1}{\left(x_{1}-x_{2}\right)^{2}} \\
\vdots \\
\frac{1}{\left(x_{n-1}-x_{n}\right)^{2}}
\end{array}\right],
$$

is replaced by the $\mathbf{E}_{\mathbf{2}}$ given by

$$
\mathbf{E}_{\mathbf{2}}(\mathbf{x}(t))=\left[\begin{array}{c}
\frac{1}{\epsilon_{1}^{2}+\left(x_{0}-x_{1}\right)^{2}} \\
\frac{1}{\epsilon_{2}^{2}+\left(x_{1}-x_{2}\right)^{2}} \\
\vdots \\
\frac{1}{\epsilon_{n}^{2}+\left(x_{n-1}-x_{n}\right)^{2}}
\end{array}\right] .
$$

As shown in Fig. 9. under this modification, the vehicle acceleration is finite when collision occurs. $\epsilon$ is fixed at 0.2 to have a maximum acceleration of $5 \mathrm{~m} . \mathrm{s}^{-2}$ which is a real vehicle parameter value.

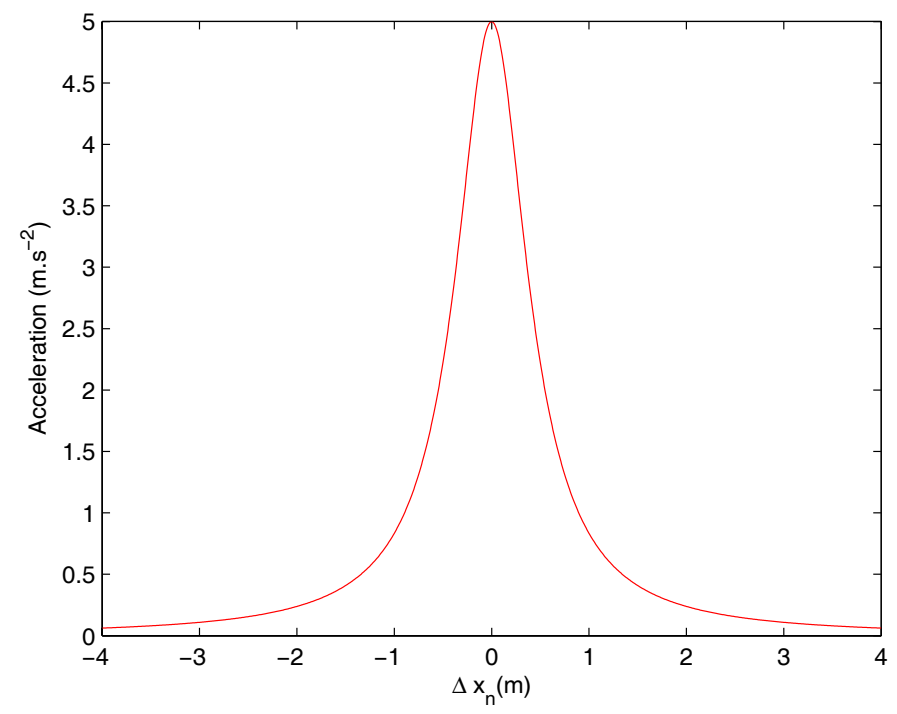

Fig. 9. Vehicle acceleration under second modification in case of collision 


\section{The full and new formulation of the Intelligent Driver Model}

\subsection{Formulation}

Assuming that the driver takes in account his follower vehicle behavior, a new parameter $h_{f}$ (human factor) is introduced in to the IDM expression. The new formulation of the Intelligent Driver Model is given by

$$
\begin{gathered}
\frac{\partial^{2}}{\partial t^{2}} x_{n}=a_{n}\left(1-\frac{\left(\frac{\partial}{\partial t} x_{n}\right)^{4}}{\nu 0_{n}^{4}}-\frac{\left(s_{n}^{*}\right)^{2}}{\epsilon^{2}+\left(\Delta x_{n}\right)^{2}}\right)+ \\
\left(h_{f}\right)_{i} a_{n-1}\left(1-\frac{\left(\frac{\partial}{\partial t} x_{n-1}\right)^{4}}{\left(v_{n-1}^{0}\right)^{4}}-\frac{\left(s_{n-1}^{*}\right)^{2}}{\epsilon^{2}+s_{n-1}^{2}}\right)
\end{gathered}
$$

where

$$
s_{n}^{*}=s_{n}^{0}+v_{n} T_{n}+\frac{c_{n} v_{n}^{2}}{b_{n}}+\frac{v_{n} \Delta v_{n}}{2 \sqrt{a_{n} b_{n}}} .
$$

For a Multiple vehicle system, the Intelligent Driver Model is given by

$$
\langle\mathbf{A}\rangle^{-1} \ddot{\mathbf{x}}(t)+\langle\mathbf{V}\rangle^{-1} \mathbf{f}_{\mathbf{1}}(\dot{\mathbf{x}}(t))+\langle\mathbf{S}\rangle \mathbf{f}_{\mathbf{2}}(x(t))=\mathbf{1}(t)+\mathbf{h}(t)
$$

with

$$
\begin{gathered}
\langle\mathbf{A}\rangle^{-1}=\left\langle\frac{1}{a_{1}}, \frac{1}{a_{2}}, \ldots, \frac{1}{a_{n}}\right\rangle, \\
\mathbf{f}_{\mathbf{1}}(\dot{\mathbf{x}}(t))=\left[\begin{array}{c}
\dot{x}_{1}^{4} \\
\dot{x}_{2}^{4} \\
\vdots \\
\dot{x}_{n}^{4}
\end{array}\right], \quad \mathbf{f}_{2}(\mathbf{x}(t))=\left[\begin{array}{c}
\frac{1}{\epsilon_{1}^{2}+\left(x_{0}-x_{1}\right)^{2}} \\
\frac{1}{\epsilon_{2}^{2}+\left(x_{1}-x_{2}\right)^{2}} \\
\vdots \\
\frac{1}{\epsilon_{n}^{2}+\left(x_{n-1}-x_{n}\right)^{2}}
\end{array}\right], \\
\mathbf{1}(t)=\left[\begin{array}{c}
1(t) \\
1(t) \\
\vdots \\
h_{1}(t) \\
h_{2}(t) \\
\vdots \\
h_{n}(t)
\end{array}\right], \mathbf{h}(t)=\left[\begin{array}{l}
(t)
\end{array}\right]
\end{gathered}
$$

with $h_{i}(t)=\left(h_{f}\right)_{i} \frac{a_{i}}{a_{i-1}} \quad \forall i=\{1 . . n-1\}$ and $h_{n}(t)=0$,

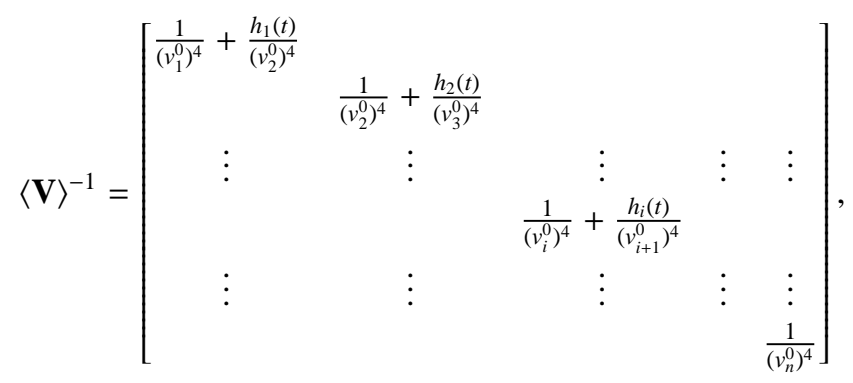

$$
\langle\mathbf{S}\rangle=\left[\begin{array}{ccccc}
\left(s_{1}^{*}\right)^{2} & \left.h_{1}(t)\right)\left(s_{2}^{*}\right)^{2} & & & \\
& \left(s_{2}^{*}\right)^{2} & h_{2}(t)\left(s_{3}^{*}\right)^{2} & & \\
\vdots & \vdots & \vdots & \vdots & \vdots \\
& & \left(s_{i}^{*}\right)^{2} & h_{i}(t)\left(s_{i+1}^{*}\right)^{2} & \\
\vdots & \vdots & \vdots & \vdots & \vdots \\
& & & & \left(s_{n}^{*}\right)^{2}
\end{array}\right] .
$$

\subsection{Determination of the parameter $h_{f}$ \\ 6.2.1 Scenario}

In this part, the modified IDM is tested with the following scenario: the platoon leader starts with a velocity of $5 \mathrm{~m} . \mathrm{s}^{-1}$. After platoon stabilization, the platoon leader speed follows a sinusoidal profile with $1 \mathrm{~Hz}$ of frequency and $1 \mathrm{~m} . \mathrm{s}^{-1}$ of amplitude. Fig. 10 shows the scenario applied in this paragraph to determine the parameter $h_{f}$.

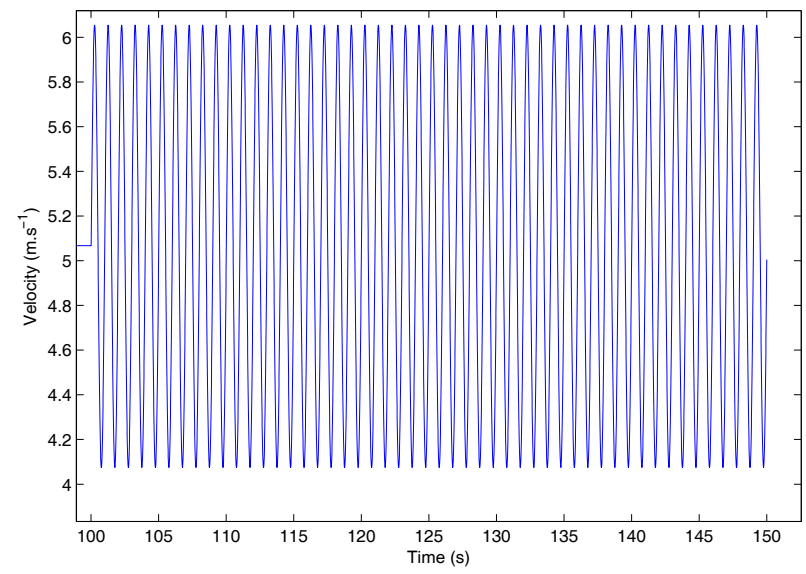

Fig. 10. Leader Speed Profile

\subsubsection{Results}

The last scenario is applied to platoon leader. This platoon is composed by seven vehicles. Fig. 11 shows the velocity of the platoon vehicle. In this figure, with $h_{f}<1$ and $h_{f}>1$, speed oscillations appears large and the relative speed between two consecutive vehicles is high. With $h_{f}=1$ oscillations is reduced and the platoon is stable.

12 show the accelerations of the platoon vehicle. In this figure, with $h_{f}<1$ and $h_{f}>1$, acceleration profile shows a large oscillations and then the relative accelerations are high. With $h_{f}=1$, each vehicle follows its leader with less oscillations and the platoon appear also stable like in Fig. 11 in term of velocities profile.

\section{Conclusions and outlook}

This paper presented the state-of-the-art of the Intelligent Driver model (IDM). Although this model was used in several research works, it presents many drawbacks. This paper presented three modifications are applied to this model. The first one consists in adding a positive term which depends on the vehicle speed, deceleration capability and a calibrated parameter. This one is determined according to an accident scenario. The optimal value of this parameter insures driver safety and the traffic flow at the same time. The second modification consists in replacing the step function by a piecewise function to insure derivative and continuity in the IDM model. The third modification consists in adding a small constant in the term of the inter-distance inside the model. This modification allow the 

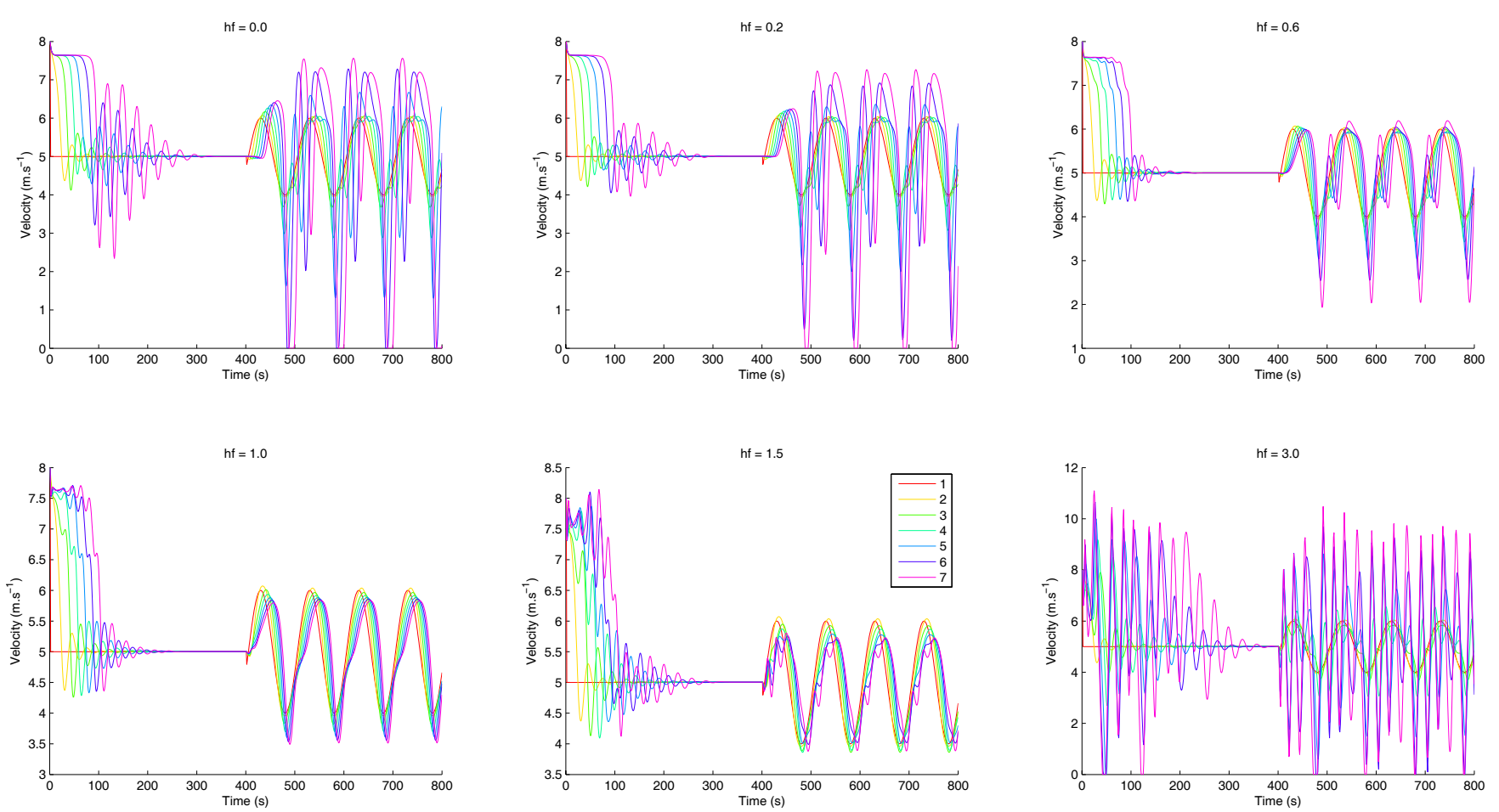

Fig. 11. Velocity of vehicles with different values of $h f$
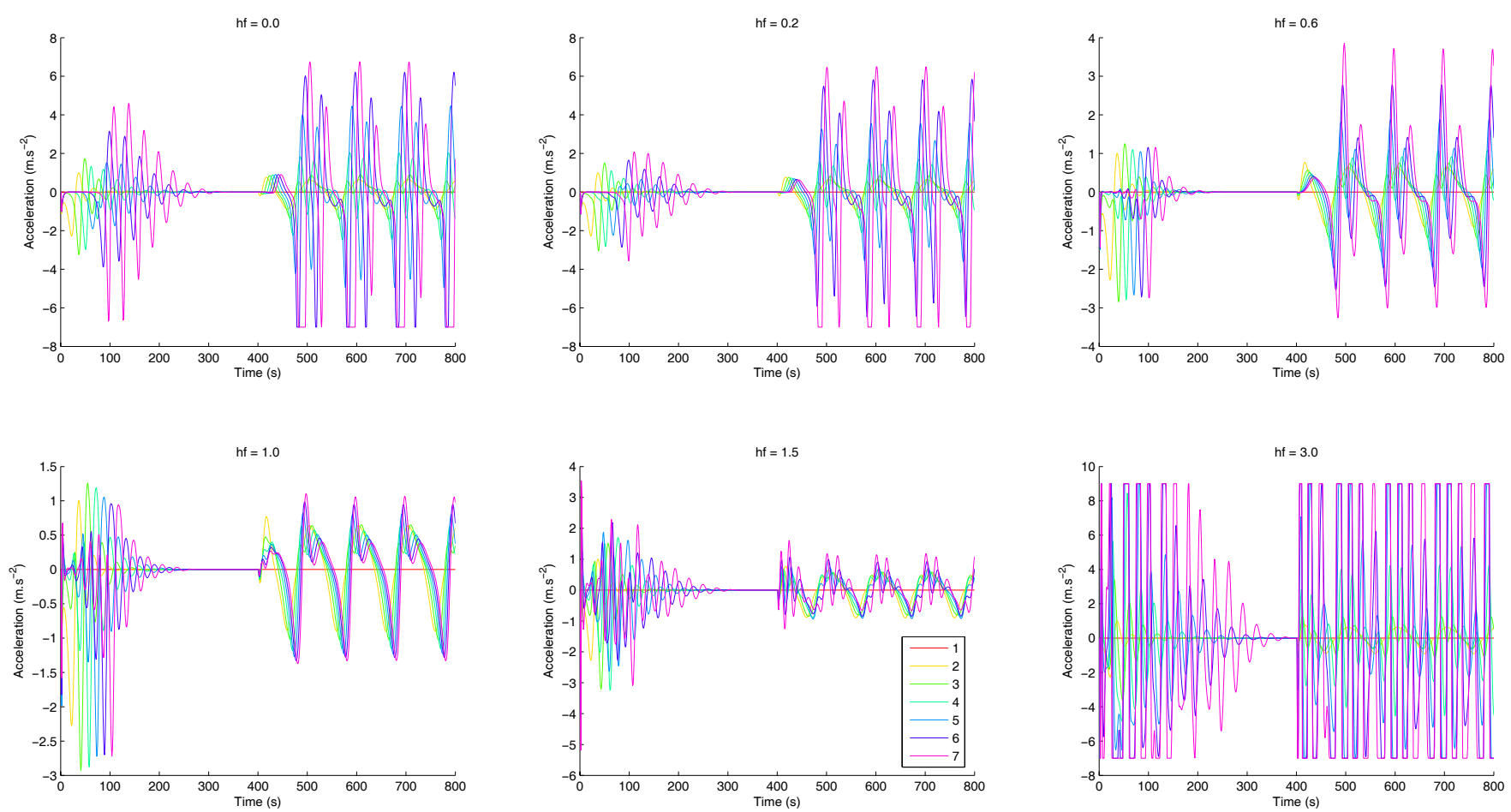

Fig. 12. Acceleration of vehicles with different values of $h f$

model to respect the vehicle's real capability in term of acceleration. Since the driver take in account the state of his follower, the fourth modification consist in taking in adding a human factor $h_{f}$ to the IDM model. As consequence, the vehicle's acceleration depends on the state of the follower and the preceding vehicle at the same time. Future works consist in implementing, into vehicle, the new IDM model and testing it in terms of driver safety and the vehicle's real capabilities.

\section{References}

1 Bede Zs, Péter T, The development of large traffic network model, Periodica Polytechnica Transportation Engineering 39 (2011), 3-6, DOI 10.3311/pp.tr.2011-1.01.

2 Bede Zs, Péter T, The mathematical modeling of Reversible Lane System, Periodica Polytechnica Transportation Engineering 39 (2011), 7-11, DOI 10.3311/pp.tr.2011-1.02.

3 Péter T, Modeling nonlinear road traffic networks for junction control, In- 
ternational Journal of Applied Mathematics and Computer Science (AMCS) 22 (2012), 723-732, DOI 10.2478/v10006-012-0054-1.

4 Bengtsson J, Adaptive Cruise Control and Driver Modeling, Lund Institute of Technology, 2001.

5 Lakatos I, Examination of effect of timing of charge replace with mathematical modell and experimentally, Acta Mechanica Slovaca 8 (2004), 403-406.

6 Lakatos I, Modern emission test of diesel engines, Europe Symposium on Euroconform Complex Retraining of Specialists in Road Transport 2001.06.09-2001.06.15. Budapest: BME, 147-153.

7 Treiber M, Helbing D, Realistische Mikrosimulation von Strassenverkehr mit einem einfachen Modell, 2002. Symposium "Simulationstechnik ASIM".

8 Treiber M, Hennecke A, Helbing D, Congested traffic states in empirical observations and microscopic simulations, Physical Review E 62 (2000), 1805-1824, DOI 10.1103/PhysRevE.62.1805.

9 Treiber M, Hennecke A, Helbing D, Delays, inaccuracies and anticipation in microscopic traffic models, Physica A: Statistical Mechanics and its Applications 360 (2006), no. 1, 71, DOI 10.1016/j.physa.2005.05.001.

10 Kesting A, Treiber M, Helbing D, Agents for Traffic Simulation, Physics and Society 17 (2011), 698-716.

11 Treiber M, Kesting A, Evidence of Convective Instability in Congested Traffic Flow: A Systematic Empirical and Theoretical Investigation, Procedia - Social and Behavioral Sciences 17 (2011), 698-716, DOI 10.1016/j.sbspro.2011.04.539.

12 Jerath K, Impact of Adaptive Cruise Control on the formation of selforganized traffic jams on Highway, The Pennsylvania State University The Graduate School. Department of Mechanical and Nuclear Engineering, 2010.

13 Derbel O, Mourllion B, Basset M, Safety assessment of mixed traffic based on accident scenario, 13-th IFAC Symposium on Control in Transportation Systems, 2012

14 Derbel O, Mourllion B, Basset M, Extended safety descriptor measures for relative safety assessment in mixed road traffic, 15th IEEE Intelligent Transportation Systems Conference (ITSC), September 2012.

15 Kesting A, Treiber M, Schonhof M, Helbing D, Adaptive cruise control design for active congestion avoidance, Transportation Research Part C: Emerging Technologies 16 (2008), no. 6, 668-683, DOI 10.1016/j.trc.2007.12.004.

16 Kesting A, Microscopic Modeling of Human and Automated Driving: Towards Traffic-Adaptive Cruise Control, Faculty of Traffic Sciences. Technische Universität Dresden (Germany), 2008. 